\title{
Steroids are part of rescue therapy in ARDS patients with refractory hypoxemia: no
}

\author{
B. Taylor Thompson ${ }^{1 *}$ and V. Marco Ranieri ${ }^{2}$
}

Rescue therapies for acute respiratory distress syndrome (ARDS) usually target patients with severe hypoxia and/ or hypercarbia refractory to conventional therapies and are considered when rapid deterioration in the patient's condition over a period of hours suggests an increased risk of death. Under these circumstances conventional mechanical ventilation will almost certainly cause additional lung injury if "rescue therapies" are not implemented. Inhaled nitric oxide, inhaled epoprostanol, high-frequency ventilation, prone positioning, or immediate cannulation for extracorporeal membrane oxygenation (ECMO) or extracorporeal carbon dioxide removal (ECCO2R) are often considerations in this setting. Three thoughtful views on the value, if any, of rescue therapies were published in Intensive Care Medicine last year [1-3]. None of these expert commentaries recommended corticosteroids as a rescue option. Should they have? Are the known effects of corticosteroids on the injured lung likely to reverse or stabilize lung injury in these catastrophically ill patients in a timely way?

When confronted with such dramatic cases clinicians should first ensure that the underlying cause of ARDS has been identified and effectively treatment started, such as appropriate antibiotics and source control for patients with sepsis and prompt management of volume overload for hypervolemic patients. Because rescue therapies are, in essence, life support or lung protective measures that do not treat the underlying disease processes leading to these catastrophic cases, intensivists must consider

\footnotetext{
*Correspondence: thompson.taylor@mgh.harvard.edu

${ }^{1}$ Division of Pulmonary and Critical Care, Department of Medicine, Massachusetts General Hospital-Harvard Medical School, 55 Fruit Street, Boston, MA 02114, USA

Full author information is available at the end of the article
}

For contrasting viewpoints, please go to doi:10.1007/s00134-015-4160-z and doi:10.1007/s00134-015-4162-x.

\section{Springer}

specific causes of ARDS or ARDS mimics that may benefit from specific therapies, including corticosteroids. ARDS mimics should be suspected when no identifiable risk factors for ARDS are apparent [4]. Examples include severe ARDS from Pneumocystis jiroveci pneumonia presenting as an AIDS-defining illness, diffuse alveolar hemorrhage from vasculitis, acute hypersensitivity pneumonitis, cryptogenic organizing pneumonia, or acute eosinophilic pneumonia. These uncommon diseases may rarely present with fulminate ARDS and have specific treatments, including corticosteroids (Table 1) [4-7].

Corticosteroids have not been systematically studied as rescue therapy for acute ARDS, so much of the evidence that bears on this question is indirect. Four randomized trials of high-dose steroids for prevention of ARDS (methyprednisolone at, for example, $30 \mathrm{mg} / \mathrm{kg}$ every $6 \mathrm{~h}$ for $24 \mathrm{~h}$, or equivalent doses dexamethazone) showed no effect or harm of this therapeutic strategy and were the subject of a contemporary Bayesean meta-analysis [8]. This analysis determined that the probability for an odds ratio of $\geq 1$ for developing ARDS and for death was 86 and $78 \%$, respectively. These probabilities suggest steroids are ineffective for prevention and probably harmful-although the credible intervals both include 1 . Accordingly, treatment with high doses of corticosteroids for short periods early in the course of critical illness has largely been abandoned. Recent meta-analyses and a systematic review of studies of lower dose corticosteroids for established ARDS show substantial heterogeneity of the pooled trials along with short-term improvement in lung physiology and outcomes, including earlier achievement of unassisted breathing $[8,9$, reviewed in 10]. Additional studies of corticosteroids for patients with ARDS and sepsis are ongoing and needed (Clinical Trials.gov identifiers NCT01731795 and NCT01448109).

Do these short-term improvements in lung physiology with corticosteroids support their use as rescue therapies? To do so, a relevant improvement of physiological variables would need to be observed in a matter of minutes or hours to "rescue" a patient from fulminant 
Table 1 Steroid-responsive conditions which may present with severe acute respiratory distress syndrome

ARDS mimics
Acute eosinophilic pneumonia (AEP)
Diffuse alveolar hemorrhage from vasculitis
Crytogenic organizing pneumonia
Acute hypersensitivity pneumonitis (HSP)
Pneumocystis jiroveci pneumonia complicating human immunodeficiency virus (HIV)
Nonspecific interstitial pneumonitis and pneumonitis associated with connective tissue disease

Some diseases, such as granulomatosis with polyangiitis leading to diffuse alveolar hemorrhage, require additional immunosupressive treatment with cyclophosphamide or rituximab [7]. Other conditions require removal of the offending antigen [heat shock proteins (HSP); asparagine endopeptidase (AEP)]. Acute interstial pneumonia (Hamman Rich) is often treated with corticosteroids but efficacy has not been established

ARDS Acute respiratory distress syndrome

ARDS. In a recently published study, Meduri et al. carefully observed the patterns of response to corticosteroids in patients with established and presumed fibroproliferative ARDS [11]. Of the 25 patients enrolled in their study, 15 demonstrated a "rapid" response to corticosteroids. Unfortunately "rapid" meant that in these responders the partial pressure of arterial oxygen/ fraction of inspired oxygen $\left(\mathrm{PaO}_{2} / \mathrm{FiO}_{2}\right)$ had improved on day 3 following initiation of steroid therapy and that static respiratory system compliance had improved on day 5 . One-third of the patients did not improve at all. Similarly, the ARDS network noted improvement in $\mathrm{PaO}_{2} / \mathrm{FiO}_{2}$ and plateau airway pressure after 3 and 4 days, respectively, of steroid therapy and more rapid liberation from mechanical ventilation [12]. Recent studies of steroids for community acquired pneumonia (CAP) also document beneficial acute responses, but the time course is relatively slow for the purposes of immediate rescue. For example, in one study of patients with severe CAP the median time to clinical stability was shorter in the steroid group [3.0 days, interquartile range (IQR) 2.5-3.4 days] than in the placebo group (4.4 days, IQR 4.0-5.0 days) [12], and in a second study of patients with CAP, time to treatment failure was reduced but the difference appeared after 4 days [13]. These encouraging data suggest corticosteroids at lower doses early in the course of pneumonia or ARDS improve lung function but that the onset of action is too slow and inconsistent and the magnitude of the effect too small to be recommended as a reliable life-saving rescue therapy. Furthermore, corticosteroids have been associated with late complications, such as secondary infections and new shock $[14,15]$.

Because of the modest, delayed, and inconsistent physiologic improvement observed with the use of corticosteroids for ARDS and CAP and the concern for late complications, we do not recommend the use of corticosteroids as rescue therapy for patients with immediately life-threatening early ARDS. Clinicians should remain vigilant for steroid-responsive diseases that may masquerade as ARDS, especially in patients without identifiable risk factors for the syndrome of ARDS. Some of these patients will require corticosteroids and other diseasespecific treatments for optimal outcomes.

\section{Author details}

1 Division of Pulmonary and Critical Care, Department of Medicine, Massachusetts General Hospital-Harvard Medical School, 55 Fruit Street, Boston, MA 02114, USA. ${ }^{2}$ Policlinico Umberto I, Anesthesia and Critical Care Medicine, "Sapienza" Università di Roma, Rome, Italy.

\section{Compliance with ethical standards}

Conflicts of interest

Both authors do not have any relevant conflicts of interest to disclose.

Received: 13 January 2016 Accepted: 28 January 2016

Published online: 16 February 2016

\section{References}

1. Coombes A, Ranieri M (2015) Rescue therapy for refractory ARDS should be offered early: yes. Intensive Care Med 41:923-925

2. Brodie D, Guerin C (2015) Rescue therapy for refractory ARDS should be offered early: no. Intensive Care Med 41:926-929

3. Roch A, Papazian L (2015) Rescue therapy for refractory ARDS should be offered early: we are not sure. Intensive Care Med 41:930-932

4. Gibelin A, Parrot A, Maitre B et al (2016) Acute respiratory distress syndrome mimickers lacking common risk factors of the Berlin definition. Intensive Care Med 42(2):164-172. doi: 10.1007/s00134-015-4064-y

5. Allen J, Pacht E, Gadek J et al (1989) Acute eosinophilic pneumonia as a reversible cause of noninfectious respiratory failure. N Engl J Med 321:569-574

6. Gagnon S, Boota AM, Fischl MA et al (1990) Corticosteroids as adjunctive therapy for severe Pneumocystis carinii pneumonia in the acquired immunodeficiency syndrome. N Engl J Med 323:1440-1450

7. Kallenberg CG (2015) Pathogenesis and treatment of ANCA-associated vasculitides. Clin Exp Rheumatol 33[4 Suppl 92]:S11-S14

8. Peter J, John P, Graham P, Moran J, Abraham I, Bersten A (2008) Corticosteroids in the prevention and treatment of acute respiratory distress syndrome (ARDS) in adults: meta-analysis. BMJ 336:1006-1009

9. Meduri GU, Bridges L, Shih MC, Marik PE, Siemieniuk RA, Kocak M (2015) Prolonged glucocorticoid treatment is associated with improved ARDS outcomes: analysis of individual patients' data from four randomized trials 
and trial-level meta-analysis of the updated literature. Intensive Care Med. doi: 10.1007/s00134-015-4095-4

10. Hough C (2014) Steroids for acute respiratory distress syndrome? Clin Chest Med 357:781-795

11. Meduri G, Chinn A, Leeper R et al (1994) Corticosteroid rescue treatment of progressive fibroproliferation in late ARDS. Patterns of corticosteroid rescue treatment of progressive. Chest 105:1516-1527

12. Steinberg K, Hudson L, Goodman R et al (2006) Efficacy and safety of corticosteroids for persistent acute respiratory distress syndrome. N Engl J Med 354:1671-1684
13. Blum C, Nigro N, Briel M et al (2015) Adjunct prednisone therapy for patients with community acquired pneumonia: a multicentre, doubleblind, randomised, placebo-controlled trial. Lancet 385:1511-1518

14. Torres A, Sibila O, Ferrer M et al (2015) Effect of corticosteroids on treatment failure among hospitalized patients with severe communityacquired pneumonia and high inflammatory response: a randomized clinical trial. JAMA 313(7):677-686

15. Sprung C, Annane D, Keh D et al (2008) Hydrocortisone therapy for patients with septic shock. N Engl J Med 358:111-124 